\title{
Cartografía de la investigación en lectura digital. Las investigaciones de Territorio Ebook en el contexto internacional
}

\section{Mapping of research in digital reading. Territory Ebook program in the international context}

\author{
Taisa Dantas \\ taisarodrigues@usal.es \\ Universidad de Salamanca. Grupo de Investigación Reconocido (GIR) E-LECTRA \\ Edición Electrónica y Lecto-escritura Digital. CAPES - Número do Processo BEX: \\ 1000144
}

\section{Raquel Goméz Díaz}

rgomez@usal.es

Universidad de Salamanca. Grupo de Investigación Reconocido (GIR) E-LECTRA Edición Electrónica y Lecto-escritura Digital CAPES - Número do Processo BEX:

1000144

\section{Resumen}

La Fundación Germán Sánchez Ruipérez realizó entre los años 2009 y 2014 una importante experiencia de investigación en el ámbito de la lectura digital: el programa Territorio Ebook. En ella estuvieron involucrados cerca de 300 lectores entre 9 y 75 años, además de bibliotecarios, autores e investigadores de la Universidad de Salamanca, Universidad Pontificia de Salamanca y Universidad de Granada. Entre los objetivos de este programa estuvo el de elaborar una etnografía del lector digital de las bibliotecas públicas de España, pero su alcance fue mayor ya que al contrastar estas investigaciones con otras hechas en diferentes países, es posible extrapolar sus resultados más allá de sus fronteras. En este artículo destaca uno de los diversos matices del programa Territorio Ebook, la investigación distribuida por grupos de edad. Se propone un estudio comparativo entre los resultados alcanzados por la Fundación Germán Sánchez Ruipérez según cada grupo de edad e investigaciones realizadas en otros lugares con esta misma variante. Como conclusión destaca la eficacia de investigar en profundidad todos los grupos de lectores, teniendo en cuenta que cada uno tiene características específicas que conducen a diferentes resultados. 


\title{
Palabras clave
}

Lectura digital, Investigación en lectura, Dispositivos de lectura, Territorio Ebook

\begin{abstract}
The Germán Sánchez Ruipérez Foundation performed between 2009 and 2014 an important research in the field of digital reading: the Territorio Ebook program. The research involved almost 300 readers, with age between 9 and 75 years, as well as librarians, authors and researchers from the University of Salamanca, Universidad Pontificia de Salamanca and the University of Granada. One of the goals of this research was the elaboration of an ethnography of the digital reader of public libraries in Spain. Nonetheless in contrasting with others researches done in different countries, it is possible to extrapolate its results beyond its borders. In this article was expose one of the various nuances of the Territory Ebook program, the research distributed by age. It is proposed a comparative study between the results achieved by the Germán Sánchez Ruipérez Foundation according to each age group and research carried out in other places with this same variant. The conclusion is the effectiveness of thoroughly investigate all groups of readers, taking into account that each has specific characteristics that lead to different results.
\end{abstract}

\section{Keywords}

Digital reading, Reading research, Reading devices, Territorio Ebook

Recibido: 15/11/2016

Aceptado: 19/12/2016

DOI: http://dx.doi.org/10.5557/IIMEI7-N13-191211

Descripción propuesta: DANTAS, Taisa; GÓMEZ DÍAZ, Raquel, 2016. Cartografía de la investigación en lectura digital. Las investigaciones de Territorio Ebook en el contexto internacional. Métodos de Información [en línea], 7(13), pp. 191-211. 


\section{Introducción}

La trayectoria seguida por los libros en los medios digitales no es reciente. Comienza con el uso de herramientas electrónicas en diferentes procesos de la creación o de la edición, hasta llegar a la etapa en que se encuentran actualmente, completamente digitales, de la escritura a la lectura (Furtado 2002; Dantas 2013; Gómez-Díaz et al. 2016). Leer en digital ya no es ninguna novedad, estamos rodeados de dispositivos electrónicos, que transforman nuestra manera de comunicar, consumir, trabajar, y, por supuesto, de leer (Nicholas 2014; Negroponte 1995; Baron 2013).

Desde el punto de vista técnico, los años 90 fueron una década muy productiva para el desarrollo del libro electrónico, con las primeras creaciones de dispositivos exclusivamente dedicados a la lectura (Cordón-García et al. 2012) hasta el punto de vista conceptual, con el despliegue de las experiencias editoriales que demostraron la interacción entre la literatura y la tecnología (Marchionne 2009), principalmente a través del uso del hipertexto en las versiones digitalizadas de las novelas publicadas originalmente en formato impreso.

Para el campo de estudio de la lectura, este desarrollo fue muy fructífero, sobre todo por el creciente interés en la realización de investigaciones sobre el impacto que la adopción de nuevas tecnologías sería capaz de generar en el hábito de la lectura. Sin embargo, esta primera fase de las investigaciones en lectura digital estaba fundamentalmente basada en los estudios comparativos entre los formatos impreso y el digital (Dillon et al. 1990).

Solamente a partir de la segunda mitad de la primera década de los años 2000 que el campo de investigación de la lectura digital empezó a crecer intensamente, asimismo su complejidad (Mangen \& van der Weel 2016). Este crecimiento es, probablemente, consecuencia del avance técnico de los dispositivos electrónicos para la lectura, que dejan de ser dispositivos experimentales, como en los años 90, y pasan a ser dispositivos de fácil manejo e uso intenso por parte de lectores comunes, como es el caso de los dispositivos Kindle (Amazon) y iPad (Apple), entre otros. 
La Fundación Germán Sánchez Ruipérez inició en 2009 una serie de investigaciones que destacan por su grado de innovación y hacen que dicha Fundación se convierta en una de las más prestigiosas y pioneras dentro del campo de estudio de la lectura y de las Tecnologías de la Información y Comunicación.

Las investigaciones del Programa Territorio Ebook, Lecturas Sin Fin, analizan el impacto de la introducción de los dispositivos de lectura electrónicos en los diferentes espacios donde los lectores leen. A partir de estos análisis ha sido posible entender los cambios que se producen en la lectura cuando se realiza con dispositivos electrónicos (e-reader y tablets) y cómo deberían diseñarse los procedimientos necesarios para implementar el uso de forma eficiente y satisfactoria de la lectura digital en las bibliotecas, aulas y centros de formación.

La motivación de la Fundación Germán Sánchez Ruipérez para realizar este tipo de investigaciones nace de una larga experiencia a lo largo de sus más de 30 años de existencia. Esta Fundación, creada en el 27 de octubre de 1981 por el editor español Germán Sánchez Ruipérez, nace de un deseo personal de su fundador. En el curso de su historia la Fundación Germán Sánchez Ruipérez amplió sus perspectivas y pasó a abarcar proyectos y programas capaces de lograr diferentes objetivos relacionados con las necesidades específicas de diferentes realidades sociales (González y Valbuena 2013), involucrando a todos los miembros de la cadena del libro, bibliotecarios, editores, autores, distribuidores y por supuesto, los lectores.

Los experimentos realizados en Territorio Ebook se llevaron a cabo desde diferentes perspectivas y están divididos en tres proyectos que se complementan entre sí: Ebook y Escuela, Ebook y Biblioteca y Ebook y Universidad. Cada proyecto buscó analizar un entorno de lectura distinto, adaptándose a estos espacios, tanto en la metodología de la investigación y los instrumentos y actividades de recopilación de datos, como en el marco teórico, actividades experimentales y análisis de resultados. Sin embargo, la principal base teórica de las investigaciones siempre estuvo enfocada en la relación entre las habilidades de lectura y las tecnologías de la información. 


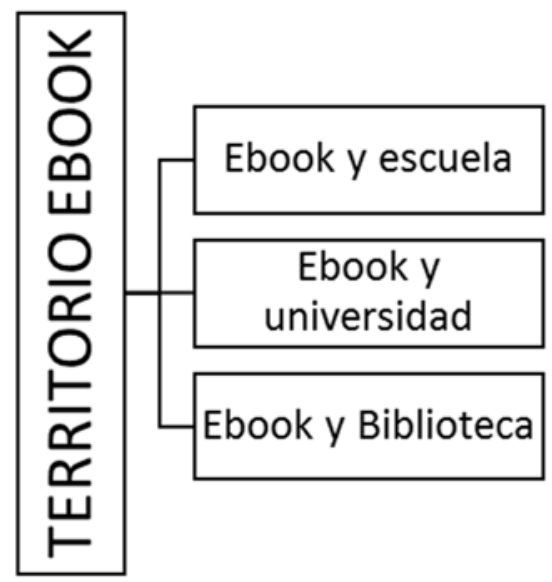

Figura 1 Proyectos integrados en Territorio Ebook

Ebook y Biblioteca fue el proyecto desarrollado por la Fundación Germán Sánchez Ruipérez para analizar cómo los lectores se relacionan con los dispositivos de lectura electrónico y las nuevas características agregadas al acto de la lectura. Este estudio sirvió para diseñar la etnografía de los lectores de las bibliotecas públicas españolas.

Estuvieron involucrados en las investigaciones del programa Territorio Ebook cuatro grupos de interés distintos. Son ellos: lectores; bibliotecarios; autores; y gestores culturales. Cada grupo representa un punto del ecosistema del libro y tuvieron papeles y actividades distintas durante toda la trayectoria de la investigación, aunque los objetivos finales se crucen.

Las actividades experimentales del proyecto Ebook y Biblioteca fueron realizadas en el Centro Internacional del Libro Infantil y Juvenil de Salamanca (CILIJ) y Centro de Desarrollo Sociocultural de Peñaranda de Bracamonte (CDS).

Dentro de este proyecto la investigación se llevó a cabo con distintos dispositivos para diferentes grupos de edad y, por tanto, con diferentes obras. Tal y como se muestra en la siguiente tabla.

Tabla 1 Proyectos incluidos dentro del proyecto Ebook y Biblioteca

\begin{tabular}{|l|l|l|l|l|l|l|l|}
\hline Fase & Edad & Fecha & Lectores & H & M & Dispositivo & Autor \\
\hline $\begin{array}{l}\text { De la } \\
\text { piedra al e- } \\
\text { book }\end{array}$ & $\begin{array}{l}+55 \\
\text { anos }\end{array}$ & $\begin{array}{l}03 / 02 / 10 \\
31 / 05 / 10\end{array}$ & 40 & 18 & 22 & Cool-er & $\begin{array}{l}\text { Luis G. } \\
\text { Jambrina }\end{array}$ \\
\hline Beatle Pad & $\begin{array}{l}13-18 \\
\text { anos }\end{array}$ & $\begin{array}{l}13 / 10 / 10 \\
16 / 12 / 10\end{array}$ & 40 & 16 & 24 & iPad & $\begin{array}{l}\text { Jordi Sierra i } \\
\text { Fabra }\end{array}$ \\
\hline $\begin{array}{l}\text { Artistas } \\
\text { Insólitos }\end{array}$ & $\begin{array}{l}9-12 \\
\text { anos }\end{array}$ & $21 / 01 / 11$ & 41 & 13 & 28 & iPad & $\begin{array}{l}\text { Daniel } \\
\text { Monedero y }\end{array}$ \\
\hline
\end{tabular}




\begin{tabular}{|c|c|c|c|c|c|c|c|}
\hline & & & & & & & Óscar Pérez \\
\hline \begin{tabular}{l}
\multicolumn{3}{l}{ Ebookeand } \\
o en la \\
nieve
\end{tabular} & $\begin{array}{l}40-54 \\
\text { anos }\end{array}$ & $25 / 02 / 11$ & 40 & 14 & 26 & $\begin{array}{l}\text { Cybook } \\
\text { Orizon de } \\
\text { Booken }\end{array}$ & $\begin{array}{l}\text { Luis G. } \\
\text { Jambrina }\end{array}$ \\
\hline $\begin{array}{l}\text { Lectores en } \\
\text { la nube }\end{array}$ & $\begin{array}{l}19-39 \\
\text { anos }\end{array}$ & $\begin{array}{l}26 / 09 / 11 \\
15 / 12 / 11\end{array}$ & 45 & 14 & 31 & $\begin{array}{ll}\text { iPad } & \text { y } \\
\text { Cybook } & \\
\text { Orizon de } \\
\text { Booken }\end{array}$ & $\begin{array}{l}\text { Lorenzo } \\
\text { Silva }\end{array}$ \\
\hline $\begin{array}{l}\text { Nube de } \\
\text { lágrimas }\end{array}$ & $\begin{array}{l}19- \\
55 \\
\text { anos }\end{array}$ & $14 / 02 / 14$ & 64 & 15 & 49 & iPad & $\begin{array}{l}\text { Rosa } \\
\text { Montero }\end{array}$ \\
\hline Total & & & 270 & 90 & 180 & 3 & 6 \\
\hline
\end{tabular}

Fuente: Basado en (González \& V albuena 2013)

Aunque las actividades experimentales fueron realizadas en las bibliotecas gestionadas por la Fundación Germán Sánchez Ruipérez, es importante destacar que todas las investigaciones fueron desarrolladas por investigadores de la Universidad de Salamanca, Universidad Pontificia de Salamanca y Universidad de Granada. Los investigadores estuvieron divididos en tres diferentes grupos, responsables por analizar perspectivas distintas de las investigaciones.

\section{Apropiación del dispositivo de lectura}

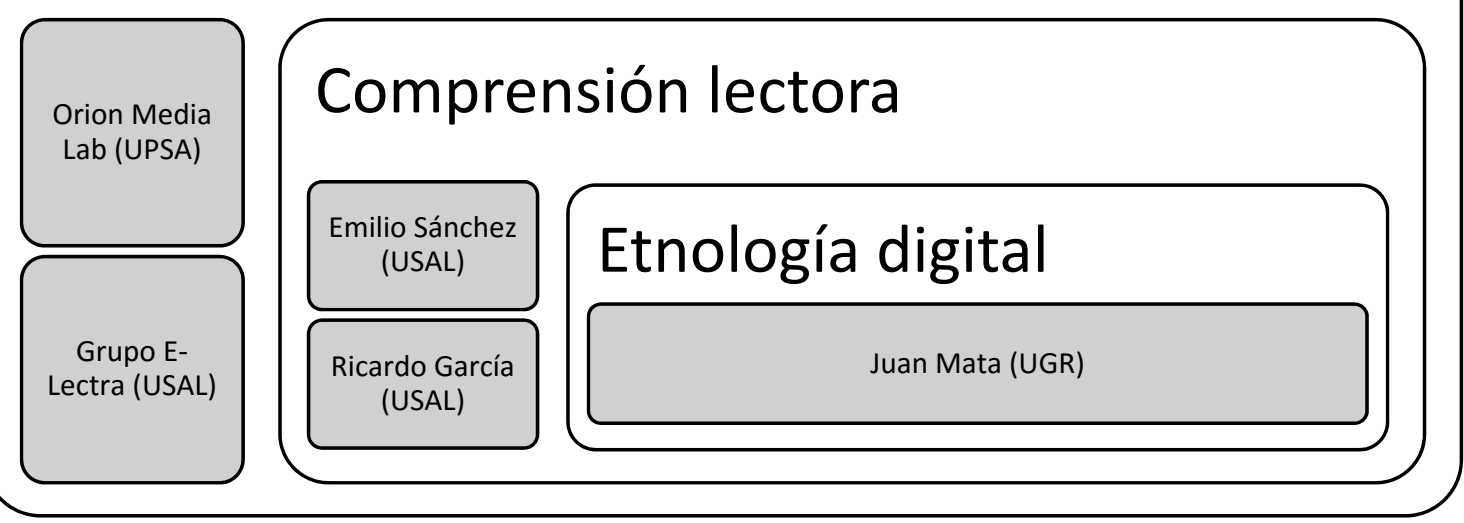

Figura 2 Principales colaboradores cientificos del programa Territorio Ebook 


\section{Objetivo}

El objetivo de este estudio es el de situar las investigaciones de la Fundación Germán Sanchéz Ruipérez dentro del escenario internacional de la investigación en lectura digital. Para ello se realiza un estudio comparativo entre los resultados obtenidos en el proyecto Ebook y Biblioteca, del programa Territorio Ebook y diversas investigaciones realizadas en otros centros de investigación o universidades.

Dada la limitación de espacio se ha elegido como criterio la variable edad, dejando otros aspectos para futuros trabajos. La elección de este indicador dentro de los estudios se debe a que resulta tremendamente complicado comparar de forma aislada estudios que proceden de ámbitos de investigación muy variados, como Psicología, Comunicación Social, Literatura, Información y Documentación, entre otro. Como son los estudios en la lectura y, por tanto, con objetivos difícilmente contrastables. La edad, sin embargo, es un elemento presente en la mayor parte de las investigaciones realizadas en lectura.

\section{Metodología}

Para el desarrollo de la investigación se han seguido varias etapas distintas. La primera ha sido la revisión bibliografica en torno a la investigación de la lectura digital en diferentes fuentes de información. Se trató de hacer la búsqueda en todos los lugares susceptibles de localizar trabajos de investigación sobre el tema, por eso se realizaron las búsquedas en bases de datos, catálogos y redes sociales de investigación.

Las bases de datos consultadas han sido tanto generales como Web of Science (Thomson Reuters), Scopus (Elsevier), y especializadas como son LISA (Library and Information Science Abstracts) y MLA (Modern Language Association of America) ambas distribuidas por ProQuest, y LISTA (Library, Information Science and Technology Abstracts) y ERIC (Education Resources Information Center) distribuidas por EBSCO. Los descriptores realizados para las búsquedas fueron "reading research" OR "reading 
investigation") OR ("investigación" Y "lectura”)], [(“digital reading” OR "digital reading research").

Asimismo, la búsqueda se completó con la búsqueda en los catálogos de la Biblioteca Nacional de España (BNE), de la Biblioteca Británica, de la Biblioteca del Congreso (EEUU), así como de la Federación Internacional de Asociaciones de Bibliotecarios y Bibliotecas (IFLA), del ISBN y de la UNESCO. También se revisaron las redes sociales de investigación ResearchGate y Mendeley.

La recopilación de los datos se realizó en enero de 2016, tomando como marco temporal de enero de 2009 hasta enero de 2016. La primera fecha fue elegida por ser el año de inicio de las investigaciones realizadas por la Fundación Germán Sánchez Ruipérez, además de ser considerado un año importante para la lectura digital, ya que se considera que el dispositivo de lectura digital Kindle (Amazon) está consolidado en el mercado y representa la transición hacia el lanzamiento de la tableta iPad (Apple).

Con estas búsquedas se recopilaron 2096 publicaciones que fueron organizadas en una base de datos elaborada en el gestor de referencias Mendeley.

La segunda etapa de esta investigación corresponde al análisis, inicialmente, de los títulos, resúmenes y palabras claves. Este análisis permitió seleccionar los estudios más relevantes, que posteriormente se analizaron con profundidad.

Posteriormente al análisis de estas publicaciones, el último paso ha consistido en realizar el comparativo entre las investigaciones realizadas en Territorio Ebook y las demás realizadas en otras partes del mundo.

\section{Análisis de resultados}

Para poder llevar a cabo la etnografía de los lectores digitales de las bibliotecas públicas, la Fundación Germán Sánchez Ruipérez dividió a los lectores en grupos de edad, que van desde los 9 a 75 años. Esta fue la forma definida por los investigadores como la más eficaz para analizar a fondo todos los grupos, teniendo en cuenta que cada uno tiene una serie de características específicas que conducen a diferentes resultados en función de la variable edad. 


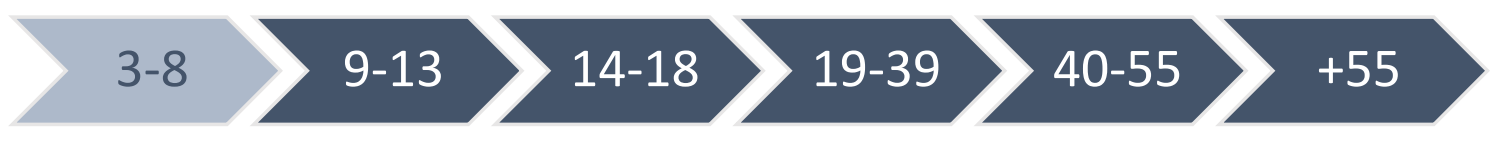

Figura 3 Segmentación por edades en los estudios de lectura

Para la discusión de los resultados se ha seguido la misma distribución por edades que los estudios de la Fundación.

\subsection{Prelectores}

Aunque la Fundación Germán Sánchez Ruipérez aún no ha abordado ninguna investigación en esta etapa, sí que se pueden encontrar trabajos que tienen como objeto de estudio los lectores de estas edades en otras investigaciones. Es importante señalar que la edad a la que los niños aprenden a leer varía de unos países a otros, incluso dentro del mismo país hay diferencias, lo que hace mucho más compleja la extrapolación de resultados.

Los estudios sobre lectura para estas edades indican que la promoción de actividades educativas relacionadas, por ejemplo, leer en voz alta, el reconocimiento de colores y números, etc., ayudan significativamente a reducir las dificultades futuras en la adquisición de la competencia en la lectura y la escritura requerida en el período de alfabetización (Justice et al. 2009).

En la actualidad, los niños en la etapa de alfabetización o pre-alfabetización (menores de 8 años) están expuestos de manera significativa a las nuevas tecnologías. Sin embargo, este es el grupo de edad donde hay menos incidencia de estudios que relacionen libros y dispositivos electrónicos. Es decir, son necesarios estudios enfocados al impacto que la exposición a libros en formatos electrónicos u otro tipo de recursos digitales relacionados con entorno del libro, pueden generar en el aprendizaje y comprensión lectora de estos futuros lectores. Asimismo, sería interesante conocer si minimizan las dificultades relacionadas con la alfabetización o si provocan distracciones que pueden hacer que ésta sea más compleja.

Los investigadores vinculados al Primate Research Institute de la Universidad de Kyoto publicaron en 2014 el resultado de una investigación dedicada a este grupo. Su trabajo tenía como objetivo entender si la narración digital de un 
libro ilustrado para niños, incluida como una de las funcionalidades de un libro electrónico, es similar a la narración realizada por un adulto a partir de un libro impreso. En este experimento participaron treinta niños de 4 años, divididos en dos grupos. El libro utilizado fue Tanabata Basu de Fujimoto (2012) y su versión digital para el iPad, donde se insertó previamente la narración digital. Un grupo utilizó la versión electrónica mientras que el otro sólo la impresa.

El principal resultado de esta investigación fue que hubo una mejora en la habilidad lectora de los participantes que utilizaron la narración digital, lo que indica que las herramientas digitales pueden ser beneficiosas para el desarrollo de la actividad de lectura (Masataka 2014).

Otra investigación también dirigida a este grupo de lectores se llevó a cabo en 2015 por investigadores de la Universidad de Columbia en los Estados Unidos. En este caso el objetivo era analizar si la relación entre las funcionalidades de los libros electrónicos, la experiencia en el entorno en el que se realizan las tareas y los conocimientos previos existentes, son capaces de generar impacto en la capacidad de comprender un formato de narrativa de cuento de hadas en formato digital (Paciga 2015). En este caso participaron en el experimento 130 alumnos con edades entre 3 y 5 años. Una característica importante presente en este estudio es que se trataba de escolares en riesgo de fracaso en la lectura, es decir, estudiantes a los cuales se esperaba que los factores económico, social o cultural, influyesen en la capacidad de adquirir habilidades relacionadas con la lectura. El experimento se realizó en dos etapas. En la primera, el investigador evaluó el conocimiento del vocabulario de cada participante y el conocimiento de la historia que sería utilizada en la investigación, así como conocimientos informáticos. Después de las actividades preliminares, los niños conocieron una las cuatro versiones del libro Stelaluna, de Cannon (1993). Cada versión tenía funcionalidades distintas, y por esto podría ser también evaluada la influencia de las habilidades en informática. Posteriormente a la lectura, los participantes expresaron verbalmente su comprensión de la narrativa.

Los resultados de esta investigación revelaron que existe una fuerte relación entre las tres variables, que en conjunto ayudan a una mejor comprensión del texto (Paciga 2015). Por lo tanto, es extremadamente positiva la interacción de los niños con los libros electrónicos, sin embargo, siempre hay que tener en cuenta su relación con los demás factores. 
A partir de los 5 años se observa una mayor incidencia de estudios dirigidos a analizar el impacto de las nuevas tecnologías en el entorno de la lectura, aunque la incidencia es aún más baja que en otros grupos de edad.

En 2012, investigadores de la Brook University realizaron un estudio con ocho niños de entre 5 y 6 años (cuatro niños y cuatro niñas) matriculados en el primer año de la enseñanza primaria en dos escuelas de la ciudad de Ontario (Canadá). Su objetivo era observar si el uso de libros electrónicos puede generar un aumento de la motivación para la lectura. La importancia de los estudios con este enfoque está relacionada con el conocimiento que la promoción de actividades de motivación entre lectores principiantes es también un factor capaz de generar impacto en el comportamiento de los lectores a lo largo de la vida (Ciampa 2012). Los experimentos se dividieron en diferentes fases compuestas principalmente de sesiones de lectura en línea. Los libros se leyeron semanalmente durante seis meses consecutivos y fueron extraídos de la sección Listening and Reading Comprehension, disponible en el sitio web Childtopia.

El principal resultado alcanzado por esta investigación fue la comprobación de que los softwares de lectura digital, junto con otros aspectos relevantes, tales como la participación de los profesores y padres y el uso del método constructivista, pueden ser responsables en el aumento de la motivación por la lectura (Ciampa 2012). Esta motivación se observó por el hecho de que los participantes tenían niveles más altos de concentración, habilidad y relación con el texto.

Dentro de la importancia del fomento de la lectura entre los lectores principiantes, posterior a la investigación realizada por la Brook University, un grupo de investigadores norteamericanos también se dedicaron a investigar este mismo grupo de edad (entre 5 y 6 años). El objetivo de su estudio fue evaluar la efectividad del uso de videojuegos de lectura digital para ayudar a la adquisición de vocabulario y habilidades de los lectores (Homer et al. 2014). El libro leído durante el experimento fue Children Make Terrible Pets de Peter Brown (2010), ya sea en forma impresa o versión digital desarrollado por Microsoft Games Studio. Esta versión fue un prototipo de juegos digitales de lectura diseñado para el videojuego Xbox 360 con Kinect (Homer et al. 2014). Para esta investigación se seleccionaron aleatoriamente 39 participantes divididos en tres grupos, donde 21 de ellos estaban inscritos en preescolar y 
18 en la escuela primaria. El primer grupo se dedicó a leer el libro impreso narrado por un adulto, mientras que en el segundo la lectura fue realizada a partir del videojuego de lectura digital, es decir, con la funcionalidad de narración digital. El tercero tuvo acceso a las mismas funcionalidades del segundo grupo, añadiendo la posibilidad de acceso a las actividades complementarias en el videojuego.

El resultado principal fue que todos los participantes alcanzaron los mismos niveles de adquisición de vocabulario, así como la motivación y la mejora de la habilidad lectora. Por lo tanto, el uso de videojuegos de lectura digital no debe ser considerado como un factor de distracción, sino más bien un instrumento útil en la promoción de la alfabetización infantil.

Todavía en el año 2013 se llevaron a cabo otros experimentos significativos dirigidos al siguiente tramo de edad: 7 y 8 años. Uno de ellos fue realizado por la Universidad de Tulsa y su objetivo era observar cómo los recursos digitales pueden ayudar en el proceso de la comprensión lectora. En este caso, se entiende por recursos digitales las herramientas de anotación, animación, diccionario, etc. (Wright et al. 2013).

Participaron en la investigación tres estudiantes matriculados en la escuela primaria, que fueron sometidos a un proceso de selección realizado por los investigadores. El principal resultado de esta investigación fue que, aunque los lectores utilizan más recursos tecnológicos, por ejemplo, el diccionario, y a pesar de que hubo un aumento del tiempo de lectura, esto no ha generado un impacto en la comprensión lectora. Sin embargo, donde sí hubo impacto fue en la motivación de lectura, ya que los lectores tenían un comportamiento más positivo en relación a esta modalidad.

Todos los estudios citados anteriormente muestran que efectivamente las nuevas tecnologías tienen un impacto positivo en la lectura entre los niños, no obstante a veces no representa un aumento directo en la comprensión, pero sí en la motivación.

Además, hay que tener en cuenta que estos estudios son preliminares, ya que son estudios con grupos extremadamente reducidos, experimentos con observaciones en un corto período de tiempo, o análisis a partir de prototipos. Variables que pueden representar distintos efectos a largo plazo o cuando la misma actividad está diseñada en diferentes situaciones. 


\subsection{Artistas Insólitos: 9 y 13 años}

El grupo con la edad más baja en las investigaciones de la Fundación Germán Sánchez Ruipérez fue el de los participantes en la tercera etapa del Ebook y Biblioteca, el experimento Artistas Insólitos (Fundación Germán Sánchez Ruipérez 2012a). En esta etapa, los participantes tenían entre 9 y 13 años de edad.

Este grupo de edad es el que tiene una incidencia más alta en las investigaciones en otras partes del mundo, dirigidas principalmente al uso de libros electrónicos o medios digitales en las actividades escolares.

La facilidad en la apropiación del dispositivo de lectura es uno de los principales factores que se destacan en este grupo de edad, lo que coincide con los lectores aún más jóvenes. Sin embargo, la principal diferencia es la capacidad crítica que tienen los participantes de esta generación.

A pesar de la rápida adaptación al dispositivo, de disfrutar de sus recursos y de que se sienten motivados para utilizarlos, la mayoría de los lectores que participaron en las investigaciones de la Fundación Germán Sánchez Ruipérez, aunque se sentían atraídos por este dispositivo, tuvieron la capacidad de tener en cuenta que por varios factores no era el dispositivo adecuado para ciertos tipos de lectura (como novelas y poesía), aunque valoraron su uso en otras actividades diarias o la lectura de otros géneros literarios.

Orientada a esta misma edad fue el trabajo realizado en Boston (EEUU) en 2013. En este experimento se trabajó con 57 estudiantes de una escuela primaria. La selección de los participantes no obedecía ningún criterio preestablecido. El objetivo de esta investigación fue observar el impacto que el uso de un dispositivo de lectura electrónica puede generar en la práctica de la lectura, motivados por el crecimiento de la tecnología centrada en la educación (Angrist 2013).

De la misma forma que las actividades promovidas por la Fundación Germán Sánchez Ruipérez, esta investigación se organizó en forma de un estudio piloto en un programa de dinamización de lectura. Durante 10 semanas se evaluaron los hábitos de lectura de los participantes. Estos participantes se dividieron en dos grupos: experimental y de control. El grupo experimental además de utilizar un dispositivo de lectura electrónica, participaba dos veces por semana de una tutoría, mientras que en el otro no hubo tutoría. 
Entre los resultados obtenidos por esta investigación, se observó la presencia de dos variables que pueden influir: el género y los factores económicos. En cuanto al género, mientras que en el caso de los niños hubo un aumento de las horas lectoras, en el de las niñas y no se detectó ninguna influencia. También se comprobó si el uso de los dispositivos electrónicos de lectura provocaba una mejora en el comportamiento de los lectores procedentes de familias con ingresos más bajos (Angrist 2013).

\subsection{BeatlePad: 14 y 18 años}

El segundo grupo de edad investigado por la Fundación Germán Sánchez Ruipérez corresponde a adolescentes con edades comprendidas entre 14 y los 18 años. Este experimento se denominó BeatlePad (Fundación Germán Sánchez Ruipérez 2012a).

Los resultados obtenidos en el experimento coinciden en muchos aspectos con los resultados obtenidos en los experimentos con el grupo de edad anterior: la gran capacidad de apropiación del dispositivo, la motivación para utilizar las funciones y los recursos disponibles y en participar de las actividades de dinamización promovidas.

Esta generación es considerada como la primera generación de nativos digitales (Prensky 2001; Piscitelli 2009), sin embargo, es una generación muy influenciada por el legado que se transmite por los responsables en orientar, fomentar y promover el hábito de la lectura, los maestros o miembros de la familia que tienen fuertes lazos con la cultura del libro impreso, y que no siempre están ni motivados ni tienen los conocimientos necesarios para hacer el acompañamiento de esos lectores.

En 2012 se publicaron los resultados de un estudio realizado por investigadores de la Universidad de Stavanger (Noruega), cuyo objetivo era precisamente el de evaluar el comportamiento de los lectores de este grupo de edad, haciendo una comparación entre el rendimiento de la lectura de textos en formato digital y la lectura de textos impresos, con el fin de evaluar cómo el formato del libro puede influir en la comprensión de la lectura (Mangen 2012).

Participaron en el experimento 72 adolescentes, con edades entre 15 y 16 años, todos matriculados en escuelas secundarias de Noruega. Los lectores fueron divididos en dos grupos, el primero de ellos hizo la lectura de dos 
textos impresos, uno narrativo y otro informativo, mientras que el segundo grupo leyó el mismo material, pero en formato digital.

El principal resultado obtenido de esta investigación fue que, efectivamente, los estudiantes que leen el texto impreso logran mejores niveles de comprensión que los que realizan la lectura en formato digital. También se observó que el género literario no influye en los resultados, pero sí el formato, y los resultados más favorables se refieren siempre a los impresos.

\subsection{Lectores en la nube: 19 y 39 años}

El siguiente grupo de edad corresponde con jóvenes y adultos, de 19 a 39 años. En el caso de las investigaciones en Territorio Ebook, representados en el proyecto Lectores en la nube. (Fundación Germán Sánchez Ruipérez 2012b). A diferencia de los grupos de edad anteriores, en este caso la franja es más amplia, y se corresponde con etapas de la vida en la que, aunque con algunas diferencias, los lectores ya están formados.

Este es el grupo de edad en el que hay más investigaciones. La mayor parte de las investigaciones se han realizado en el entorno universitario.

En 2009 se dieron a conocer los resultados de una investigación realizada entre los años de 2006 y 2008 en la Chinese University of Hong Kong. A pesar de que corresponde a un estudio centrado en la eficiencia del uso de los dispositivos de lectura electrónico, este estudio es similar al el de la Fundación Germán Sánchez Ruipérez, principalmente en las secciones dedicadas al análisis de la capacidad de apropiación de los lectores.

El estudio estuvo motivado por la constatación de que la evolución de los medios digitales ya era una realidad. Acceder a contenido en línea se había convertido en una actividad cotidiana, sin embargo, la eficiencia de los dispositivos para acceder a los materiales de estudio digitales todavía era desconocida. Por lo tanto, era imprescindible realizar un estudio que tratara de comprender si los estudiantes estaban o no preparados para el uso de este soporte y cuáles son las fortalezas y debilidades encontradas en este entorno. Participaron en la investigación 24 estudiantes, que fueron divididos en dos grupos. Un grupo recibió una introducción al dispositivo de lectura y luego fueron motivados para que comentasen sus primeras impresiones de la tecnología. Mientras tanto, el segundo grupo participó en un experimento de seguimiento de 12 semanas de duración, en el que la lectura digital realizada 
fue supervisada por los investigadores (Lam et al. 2009) y fue, en cierto modo, similar a la realizada en Territorio Ebook.

Entre los resultados obtenidos por la investigación es importante resaltar la visión de los investigadores en percibir que los dispositivos de lectura electrónica tienen el potencial de ser utilizado para el aprendizaje y la enseñanza, sin embargo, todavía hay varias dificultades que superar. Las dificultades destacan cuando se trata del grupo que participó en la experiencia de seguimiento. Mostrando que, aunque de manera independiente es fácil aprender a utilizar los dispositivos electrónicos, a largo plazo, se pierde la motivación por la lectura. La facilidad en la apropiación se da principalmente porque es un grupo de edad considerado inmigrante digital, pero tiene un fuerte acceso a los dispositivos tecnológicos.

En 2013 los investigadores vinculados a la Bar-Ilan University y a la Open University of Israel realizaron un estudio con 93 estudiantes con una edad media de 23 años, matriculados en el área de las Ciencias Sociales.

La investigación tenía como objetivo observar las diferencias entre la lectura digital y la lectura impresa, realizando un análisis centrado en las capacidades que los estudiantes para ejecutar una lectura activa, es decir, la elaboración de notas, edición, reconocimiento de errores y de la calidad del texto (Eden \& Eshet-Alkalai 2013).

La actividad experimental fue la lectura activa de un texto durante 20 minutos, seguido de un cuestionario elaborado por los investigadores. Destacamos en los resultados que hubo una adaptación extremadamente positiva a este tipo de lectura digital. "Surprisingly, and in contrast to the common reported findings from print versus digital reading studies, no significant differences were found between the performances of participants in the two formats." (Eden \& Eshet-Alkalai 2013).

Las investigaciones con lectores en la misma franja de edad realizadas por la Fundación Germán Sánchez Ruipérez, aunque no se trata de una comparación entre lo digital y lo impreso, se alcanzaron resultados similares, dado que no hubo rechazo a la lectura digital.

En cuanto a las dos siguientes franjas de edad investigadas por la Fundación Germán Sánchez Ruipérez, entre 40 y 55 años, y más de 55 años, no hay ocurrencia de otros estudios relacionados con el campo de la investigación de la lectura digital. 
El gran aporte de las investigaciones con lectores mayores de 55 años que participaron en el proyecto De la piedra al Ebook (Fundación Germán Sánchez Ruipérez 2011), ha sido el de comprobar que, aunque se trate de una generación en la que toda su educación y experiencia lectora ha sido basada en los formatos impresos, la adaptación a los formatos digitales es excelente. Esto comprobaba la hipótesis de que un lector digital no nace, se forma, aunque sus orígenes sean analógicos.

Esta misma hipótesis fue confirmada, en cierto grado, en los otros dos proyectos que involucraron adultos Ebookeando en la nieve (Fundación Germán Sánchez Ruipérez 2013) y Nube de Lágrimas (Fundación Germán Sánchez Ruipérez 2014). Sin embargo, en determinados momentos, esta generación inmigrante, diferente de los mayores de 55 años, dejaron su acento analógico (Prensky 2001) influir. Una vez que en determinados momentos de las investigaciones fue notado vestigios de prejuicio y resistencia a los dispositivos de lectura, a pesar de la facilidad en la apropiación.

\section{Conclusiones}

El análisis de todos estos trabajos permite por un lado mostrar como en los últimos años los principales temas que se han tratado han sido:

- La apropiación de los dispositivos de lectura.

- Impacto del uso de los dispositivos de lectura en el fomento a la lectura y en el entorno educativo.

- Las nuevas características relacionadas con la lectura digital.

- Comparación entre la lectura en soportes digitales e impresos.

La suma de las investigaciones hechas hasta el momento de la recopilación de datos ha originado los siguientes resultados:

- La motivación a la lectura es uno de los principales aportes del uso de los dispositivos electrónicos.

- Todavía existe una gran preocupación acerca de la apropiación de los dispositivos de lectura y de los efectos negativos que pueden generar. 
- Aunque la lectura digital tenga características propias, pocas son las investigaciones centradas solamente en esta modalidad de lectura.

- El entorno educativo es el espacio en que más se centran las investigaciones en la lectura.

- Independientemente del rango de edad todos los lectores son propensos a ejecutar positivamente la lectura digital.

Además, el estudio de estos trabajos y su comparación con la Fundación Germán Sánchez Ruipérez permite poner en valor la labor investigadora que durante cuatro años se realizó, ya que ninguna otra institución ha liderado investigaciones con tantos grupos de edad (salvo en la primera franja la fundación ha investigado con todas las edades) ni se ha mantenido en el tiempo.

\section{Referencias}

ANGRIST, N., 2013. Read alert: The effects of eBooks on reading outcomes: evidence from a randomized trial in Boston. SSRN Electronic Journal. ISSN 15565068. doi 10.2139/ssrn.2298645.

BARON, N.S., 2013. Redefining reading: The impact of digital communication media. Publicacions of the Modern Language Association of America, 128 (1), 193 200. doi 10.2307/23489277.

CIAMPA, K., 2012. Electronic storybooks: A constructivist approach to improving reading motivation in grade 1 students. Canadian Journal of Education, 35 (4), 92-136. ISSN 03802361.

DANTAS, T.R., 2013. The digital reading as a product of the evolution of information: Books between screens. En: 1st International Conference on Technological Ecosystem for Enhancing Multiculturality, TEEM 2013. 14-15 noviembre. Salamanca. ACM. 375-379. ISBN 9781450323451. doi $10.1145 / 2536536.2536593$.

DILLON, A., RICHARDSON, J. y MCKNIGHT, C., 1990. The effects of display size and text splitting on reading lengthy text from screen. Bebaviour and Information Technology, 9(3), 215-227 
EDEN, S. y ESHET-ALKALAI, Y., 2013. The effect of format on performance: Editing text in print versus digital formats. British Journal of Educational Technology, 44 (5), 846-856. ISSN 00071013. doi 10.1111/j.1467-8535.2012.01332.x.

FUNDACIÓN GERMÁN SÁNCHEZ RUIPÉREZ, 2011. Ebook 55+ Los lectores mayores de 55 años y los libros electrónicos. [en línea]. Peñaranda de Bracamonte. Consulta: 05 noviembre 2016. Disponible en: http:/ / es.calameo.com/read/00050956333174ff88d82.

FUNDACIÓN GERMÁN SÁNCHEZ RUIPÉREZ, 2012b. Ebook -18. Los lectores niños y jóvenes y los libros electrónicos. [ en línea]. Peñaranda de Bracamonte. Consulta: 05 noviembre 2016. Disponible en: http:/ / pt.calameo.com/read/0005095631d2ce80a7ad1

FUNDACIÓN GERMÁN SÁNCHEZ RUIPÉREZ, 2013. Ebook +40 -55 - Los lectores adultos y los libros electrónicos. [en línea]. Peñaranda de Bracamonte. Consulta: 05 noviembre 2016.2 Disponible en: http://pt.calameo.com/books/000509563722d70890f18

FUNDACIÓN GERMÁN SÁNCHEZ RUIPÉREZ, 2014. Nube de Lágrimas. Club de lectura en la nube. Lectores digitales y lectura social. [ en línea]. Peñaranda de Bracamonte. Consulta: 05 noviembre 2016. Disponible en: http:/ / pt.calameo.com/read/00050956329f4617050ec

FURTADO, J.A., 2002. Livro e leitura no novo ambiente digital. [en línea]. Consulta: 01 noviembre 2016.2 Disponible en: http://www.moodle.ufba.br/file.php/10203/Textos_Topicos/leituraescrita/ Livro_e_leitura_no_novo_ambiente_digital.pdf.

GARCÍA-CORDÓN, J.A., DÍAZ-GÓMEZ, R. y ARÉVALO-ALONSO, J., 2012. Gutenberg 2.0. La revolución de los libros electrónicos. Gijón.Trea. ISBN 97884-9704-552-0.

GÓMEZ-DÍAZ，R., GARCÍA-RODRÍGUEZ，A., CORDÓN-GARCÍA, J.A. y ALONSO-ARÉVALO, J., 2016. Leyendo entre Pantallas. Gijón. Trea. ISBN 978-84-9704-945-0. 
GONZÁLEZ, L. y VALBUENA, J., 2013. A prueba de futuro: la investigación de la Fundación Germán Sánchez Ruipérez. En: J.A GARCÍACORDÓN et al coor. Libros electrónicos y contenidos digitales en la sociedad del conocimiento: mercado, servicios y derechos. Madrid: Pirámide, pp. 305-323. ISBN 978-84-368-2769-9

HOMER, B.D., KINZER, C.K., PLASS, J.L., LETOURNEAU, S.M., HOFFMAN, D., BROMLEY, M., HAYWARD, E.O., TURKAY, S. y KORNAK, Y., 2014. Moved to learn: The effects of interactivity in a Kinectbased literacy game for beginning readers. Computers \& Education. 74 (mayo) 37-49. ISSN 03601315. doi 10.1016/j.compedu.2014.01.007..

JUSTICE, L.M., KADERAVEK, J.N., FAN, X., SOFKA, A. y HUNT, A., 2009. Accelerating preschoolers' early literacy development through classroom-based teacher-child storybook reading and explicit print referencing. Language, speech, and hearing services in schools. 40 (1), 67-85. ISSN 0161-1461. doi 10.1044/0161-1461(2008/07-0098).

LAM, P., LAM, S.L., LAM, J. y MCNAUGHT, C., 2009. Usability and usefulness of eBooks on PPCs: How students' opinions vary over time. Australasian Journal of Educational Technology. 25 (1), 30-44. ISSN 14495554. doi 10.1177/1080569911432628.

MANGEN, A., 2012. Literary reading on paper and screen: An experiment comparing narrative immersion on paper and iPad. En: IGEL: The International Society for the Empirical Study of Literature and Media. 09 julio. Montreal.

MANGEN, A. y VAN DER WEEL, A., 2016. The evolution of reading in the age of digitisation: an integrative framework for reading research. Literacy. 50 (3) 116-124. ISSN 17414350. doi 10.1111/lit.12086.

MARCHIONNE, I., 2009. Il ruolo della critica letteraria nella rivoluzione digitale. Il caso della letteratura italiana. Università degli Studi di Firenze.

MASATAKA, N., 2014. Development of reading ability is facilitated by intensive exposure to a digital children's picture book.Frontiers in Psychology. 5 (mayo) 5-8. ISSN 16641078. doi 10.3389/fpsyg.2014.00396. 
NEGROPONTE, N., 1995. El mundo digital. Barcelona: Ediciones B. ISBN 8440659253.

NICHOLAS, D., 2014. The Google generation, the mobile phone and the «library» of the future: Implications for society, governments and libraries. En: A. NOORHIDAW ATI et al, eds. ICOLIS. Kuala-Lumpur. DLIS, FCSIT .1-8. Consulta en 07 noviembre 2016. Disponible en: http://ciberresearch.eu/download/20141105-Malaysia_Nicholas_keynote.pdf

PACIGA, K.A., 2015. Their teacher can't be an app: Preschoolers' listening comprehension of digital storybooks. Journal of Early Childhood Literacy.15(4) 473-509. ISSN 1468-7984. doi 10.1177/1468798414552510.

PISCITELLI, A., 2009. Nativos digitales: dieta cognitiva, inteligencia colectiva y arquitecturas de la participación. Buenos Aires. Santillana. ISBN 9789504621317.

PRENSKY, M., 2001. Digital Natives, Digital Immigrants, Part II: Do They Really Think Differently? On the Horizon. 9(6), 9. [Consulta: 15 octubre 2016. Disponible en: http://www.marcprensky.com/writing/Prensky - Digital Natives, Digital Immigrants

Part2.pdf \nhttp://www.bucks.edu/media/thinkdifferently_prensky.pdf $\backslash$ nhtt p://eventos.unipampa.edu.br/seminariodocente/files/2011/03/Oficina-11Prensky-Digital_natives_Digital.

WRIGHT, S., FUGETT, A. y CAPUTA, F., 2013. Using E-readers and internet resources to support comprehension. Educational Technology and Society. 16 (1), 367-379. ISSN 11763647. doi 10.2307/jeductechsoci.16.1.367. 
